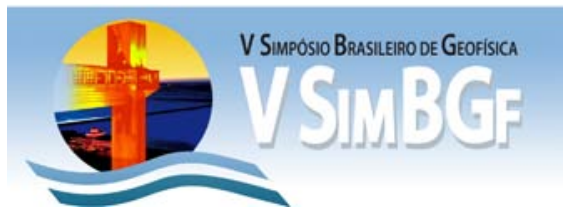

\title{
Parametrização de configuração de cargas em sísmica 2D terrestre
}

Joelson da Conceição Batista ${ }^{1}$, Marcelo Ferreira de Araújoํㅜ, 1 - Georadar Levantamentos Geofísicos S/A.

Copyright 2012, SBGf - Sociedade Brasileira de Geofísica

Este texto foi preparado para a apresentação no V Simpósio Brasileiro de Geofísica, Salvador, 27 a 29 de novembro de 2012. Seu conteúdo foi revisado pelo Comitê seus associados. É proibida a reprodução total ou parcial deste material para propósitos comerciais sem prévia autorização da SBGf.

\section{Resumo}

A escolha dos parâmetros de aquisição sísmica tem influência direta na qualidade dos dados adquiridos e no mapeamento das estruturas em subsuperfície. Uma aquisição mal parametrizada conduzirá a dados sísmicos com baixa razão sinal/ruído e baixa multiplicidade o que pode comprometer seriamente a qualidade das imagens de subsuperfície geradas como resultado final do processamento sísmico. No presente trabalho investigamos o desempenho de dois arranjos de cargas. Um primeiro formado por 3 cargas de $1 \mathrm{~kg}$ espaçadas por $10 \mathrm{~m}$ e um segundo por 2 cargas espaçadas de $20 \mathrm{~m}$ com $1 \mathrm{~kg}$ cada. Essas cargas ficaram a uma profundidade de $3 \mathrm{~m}$ da superfície.

\section{Introdução}

O presente trabalho faz uma comparação dos resultados obtidos através do levantamento de campo utilizando fontes de energia do tipo dinamite arranjadas em duas configurações distintas. O estudo foi realizado com dados de uma região com geologia conhecida cedidos pela OGX Petróleo e Gás Ltda. e a Petra Energia.

O objetivo deste trabalho é mostrar uma análise do sinal sísmico usando dados adquiridos com diferentes arranjos de carga abordando as vantagens e desvantagens quanto à qualidade final de processamento.

O depocentro da sequência sedimentar atinge espessuras maiores que $3000 \mathrm{~m}$. O seu substrato é constituído por rochas metamórficas, ígneas e sedimentares. O embasamento é constituído por duas unidades sedimentares compostas de grauvacas, arcósios, siltitos e folhelhos.

A escolha da energia da fonte depende grandemente da profundidade do horizonte de interesse, enquanto que sua profundidade está ligada à tentativa de eliminar a influência de camadas superficiais intemperizadas. Dessa forma, devemos considerar algumas observações sobre redução de furos e cargas:

- A carga e a profundidade são dimensionadas de forma que na profundidade do alvo, exista uma máxima relação sinal/ruído (S/R).

- Alvos profundos requerem geralmente maior energia e nesse sentido, caso a profundidade seja inadequada para a carga dimensionada, no momento de sua detonação poderá ocorrer o colapso do furo que poderá emitir material em todas as direções, o que pode ocasionar danos a estruturas e pessoas na vizinhança do levantamento, fazendo com que grande parte dessa energia seja perdida sob forma de ondas aéreas, gerando concomitantemente ruídos espúrios no dado (groundroll).

- Geralmente cargas com grande energia geram maior contaminação com groundroll e de ondas aéreas nos registros. Alta energia produz um conteúdo de baixa frequência dominante, porém uma distribuição de frequência mais ampla se comparado com o uso de cargas com baixa energia. O uso de baixa energia pode produzir o predomínio do conteúdo de alta frequência, mas com uma distribuição menos uniforme.

O processamento de dados sísmicos de reflexão é uma atividade de rotina na indústria do petróleo e tem por objetivo a obtenção de imagens sísmicas representativas da geologia de subsuperfície. Nesse sentido, a qualidade dos dados sísmicos desempenha um papel fundamental e a presença do groundroll nos dados sísmicos terrestres é, por conseguinte, um fator que compromete seriamente a qualidade final do processamento. Uma série de filtragens e manipulações pode ser aplicada com essa finalidade de atenuar os ruídos, em duas etapas básicas: (i) o pré-processamento que compreende a preparação e o tratamento dos dados através de técnicas simples de filtragens e (i) o processamento avançado, que envolve a aplicação de técnicas um pouco mais sofisticadas (deconvolução, filtragem multicanal FK, balanceamento espectral, DMO, migração). Todas as etapas realizadas durante 0 processamento compõem o chamado fluxograma de processamento sísmico.

O groundroll ocorre na forma de um cone de ruído com reflexões lineares, de baixa freqüência e alta amplitude, que se superpõe mascarando as demais reflexões, prejudicando seriamente a razão sinal/ruído dos sismogramas. A remoção ou atenuação do groundroll é necessária para a obtenção de seções sísmicas de boa qualidade. Os métodos de filtragem monocanais simples (e.g. filtro de freqüência passa-banda) e multicanais (e.g. filtro FK) podem ser utilizados com essa finalidade, apesar de terem a desvantagem de também atenuarem o sinal.

Esse estudo faz um comparativo dos registros sísmicos de três linhas testes, em relação ao conteúdo de freqüência, nível de atenuação do groundroll, envelope de amplitude dos registros e continuidade da primeira quebra de acordo com o arranjo de carga utilizado. Foram utilizados dois arranjos. O arranjo de cargas 1, formado por um conjunto de três cargas, onde duas 
cargas laterais permaneceram distantes $10 \mathrm{~m}$ da carga central e o arranjo de cargas 2, formado por duas cargas espaçadas de $20 \mathrm{~m}$. Nesses dois arranjos cada carga possuía $1 \mathrm{~kg}$ e a profundidade da fonte foi de 3 metros. $\mathrm{Na}$ linha teste 1 foi usado o arranjo com três cargas (arranjo de cargas 1), na linha teste 2 o arranjo com 2 cargas (arranjo de cargas 2) e na linha teste 3 foi feita a intercalação desses dois arranjos para efeito de processamento.

A tabela abaixo mostra de forma mais detalhada os parâmetros de aquisição das linhas.

\begin{tabular}{|c|c|}
\hline \multicolumn{2}{|c|}{ Parâmetros de Aquisição } \\
\hline Número de canais & 320 \\
\hline Intervalo entre tiros & $25 \mathrm{~m}$ \\
\hline $\begin{array}{c}\text { Intervalo entre estações } \\
\text { de receptores }\end{array}$ & $25 \mathrm{~m}$ \\
\hline Cobertura & 80 \\
\hline Tempo de registro & $5 \mathrm{~s}$ \\
\hline Razão de amostragem & $2 \mathrm{~s}$ \\
\hline Profundidade de carga & $3 \mathrm{~m}$ \\
\hline Arranjo de cargas 1 & $3 \mathrm{~kg}$ \\
\hline Arranjo de cargas 2 & $2 \mathrm{~kg}$ \\
\hline
\end{tabular}

Tabela 1 - Parâmetros de aquisição

\section{Análise do sinal}

As figuras 1 e 2 exemplificam qualitativamente a qualidade dos dados utilizados.

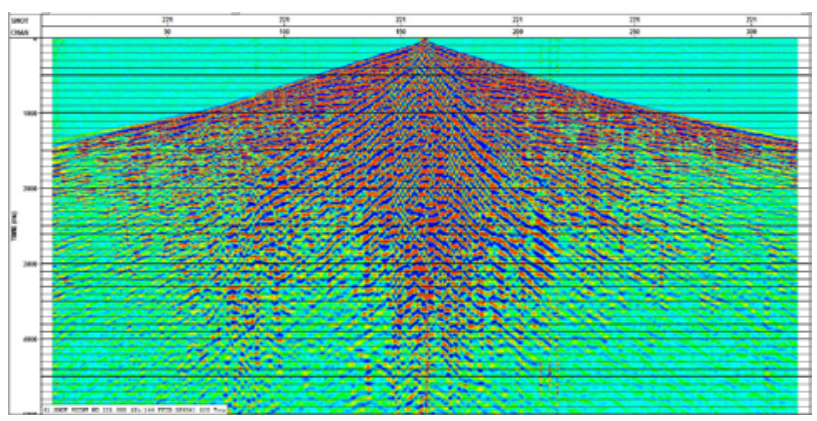

Figura 1 - Sismograma da linha teste 1.

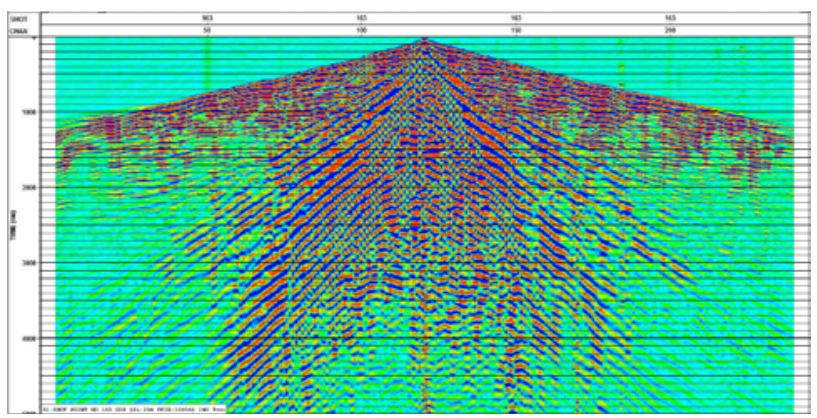

Figura 2 - Sismograma da linha teste 2.

As figuras 3 e 4 representam o envelope de amplitude de energia registrada nos dados adquiridos pela linha teste 1 e 2, respectivamente. Observa-se que parte da energia registrada no teste 2 concentra-se fortemente no cone do groundroll e que o teste 1 apresentou uma distribuição mais homogênea dessa energia ao longo de todo o offset.

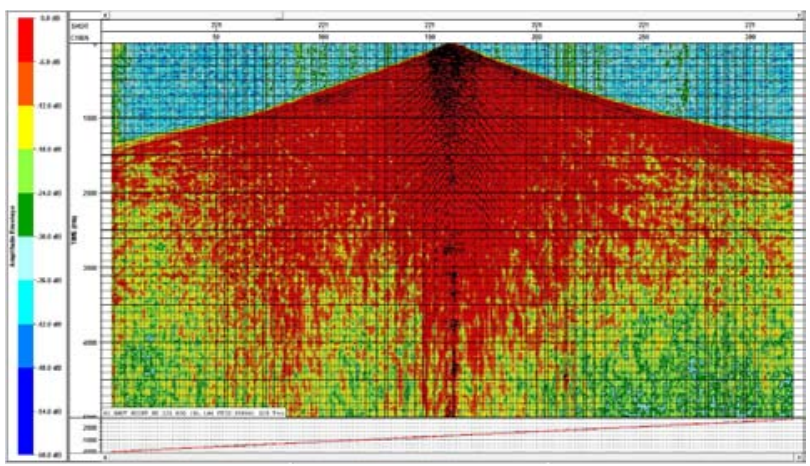

Figura 3 - Envelope de amplitude - linha teste 1.

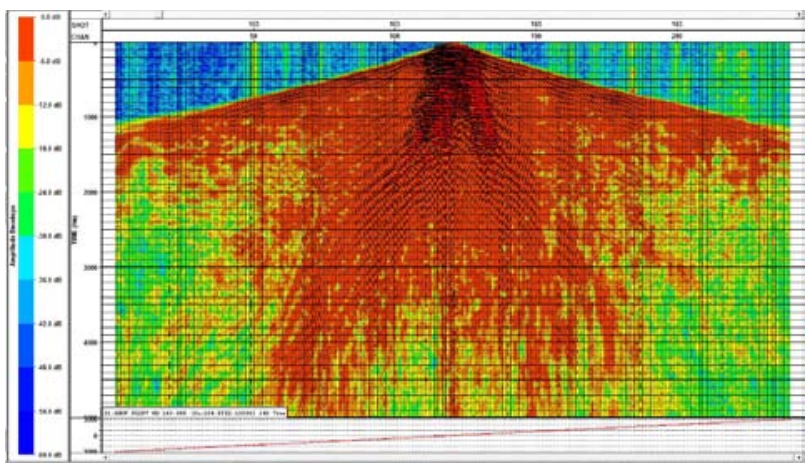

Figura 4 - Envelope de amplitude - linha teste 2.

De acordo com os gráficos das figuras 5 e 6 , as faixas de frequência com valores mais elevados de $S / R$ são as de 0/05/15/20 Hz, 10/15/25/30 Hz, 20/25/35/40 Hz e $30 / 35 / 40 / 50 \mathrm{~Hz}$. Em média a S/R é melhor nos dados da linha teste 2. Esse demonstrou bons valores de S/R na faixa de 0/05/15/20 Hz, entretanto esses valores estão concentrados no cone do groundroll, o que pode ser observado quando comparamos as figuras 7 e 8 . Ainda nas figuras 5 e 6 , as faixas de frequência de 40/45/55/60 $\mathrm{Hz}$ e 50/55/65/70 Hz tem valores baixo de S/R exceto nas porções mais rasas, onde o teste 1 foi ligeiramente maior. As figuras 9 e 10 representam a amplitude da S/R para os dados das linhas testes 1 e 2, reforçando as características citadas. Neste caso o teste 2 apresenta elevados valores de amplitude que decai fortemente com o aumento da freqüência, o que não acontece com o teste 1. 


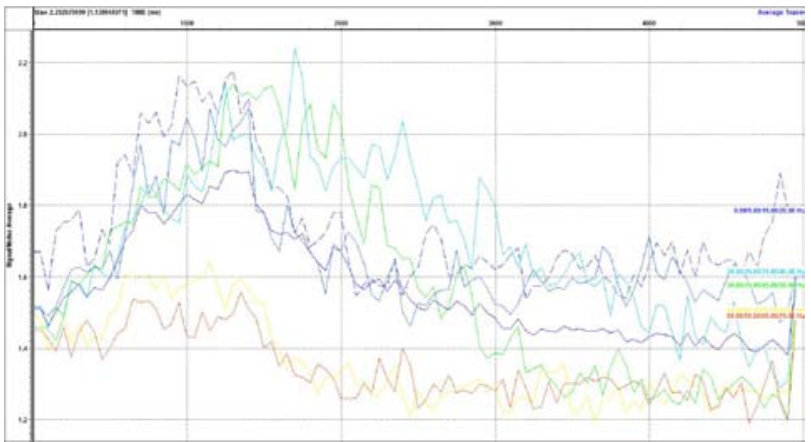

Figura 5 - Razão sinal/ruído em função da frequência teste 1.

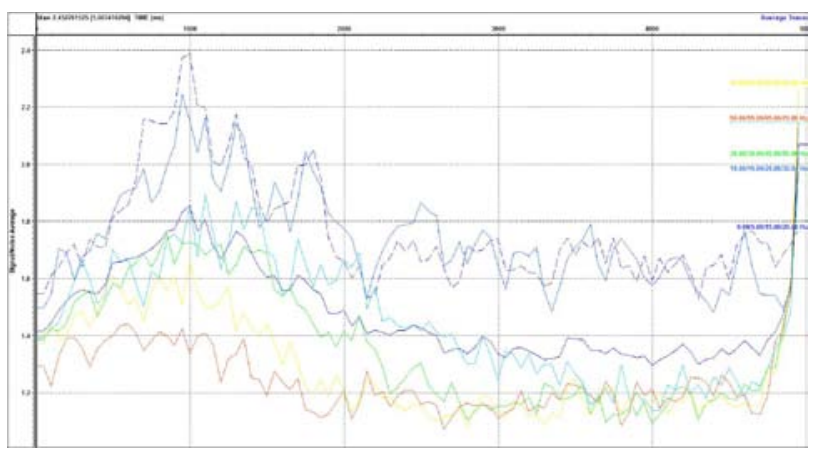

Figura 6 - Razão sinal/ruído em função da frequência teste2.

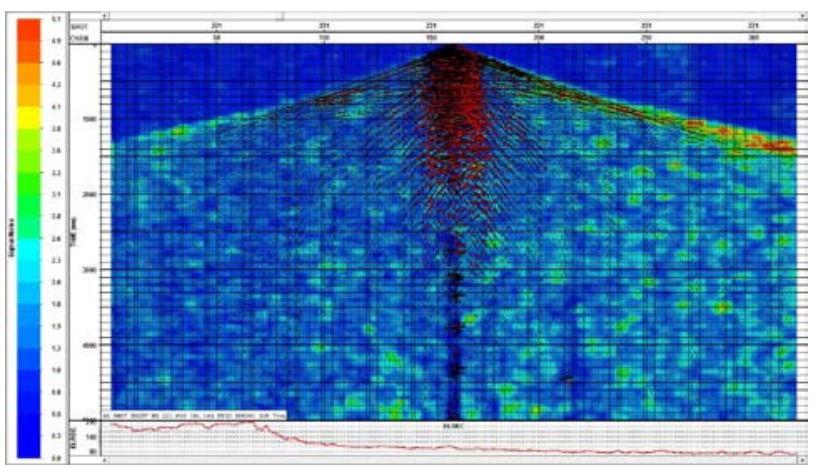

Figura 7 - Razão sinal/ruído - teste 1.

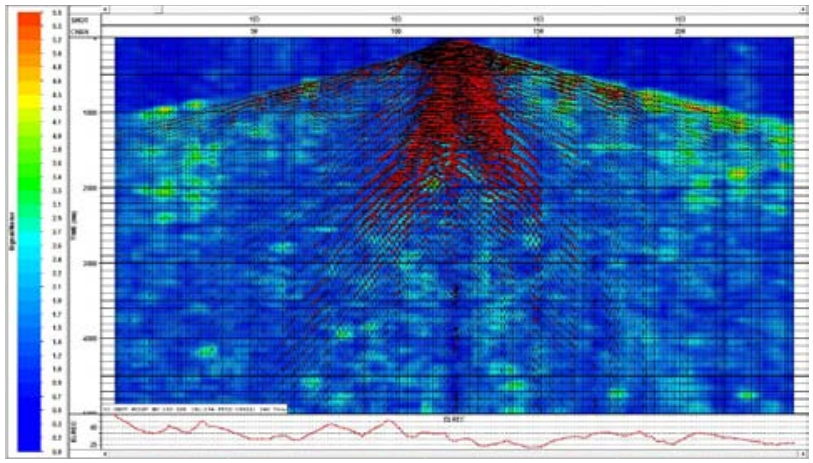

Figura 8 - Razão sinal/ruído - teste 2.

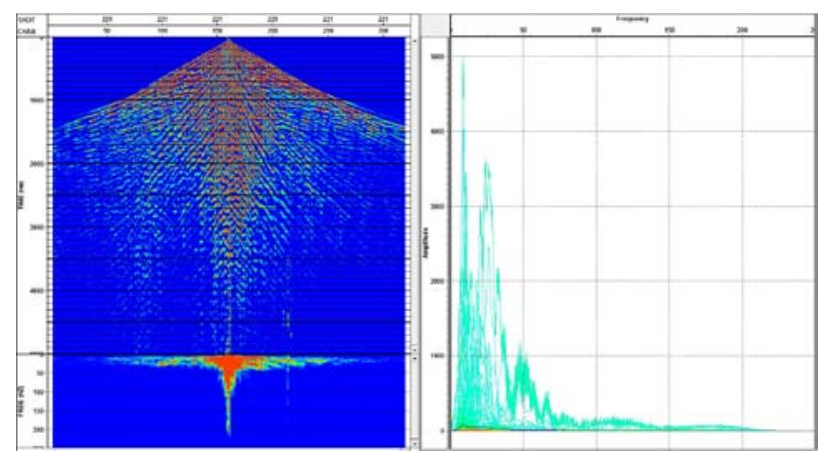

Figura 9 - Analise de frequência do sinal - teste 1.

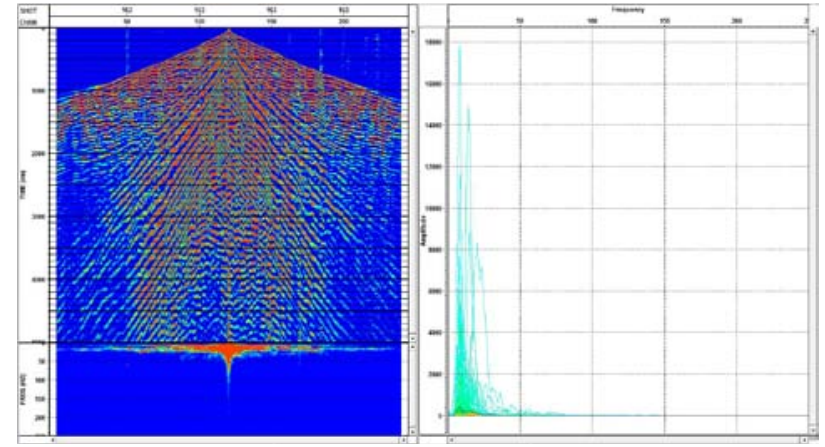

Figura 10 - Analise da frequência - teste 2.

\section{Processamento}

As linhas com diferentes arranjos de cargas, denominadas linhas testes, serão submetidas à sequência básica de processamento, conforme fluxograma exemplificado na figura 11.

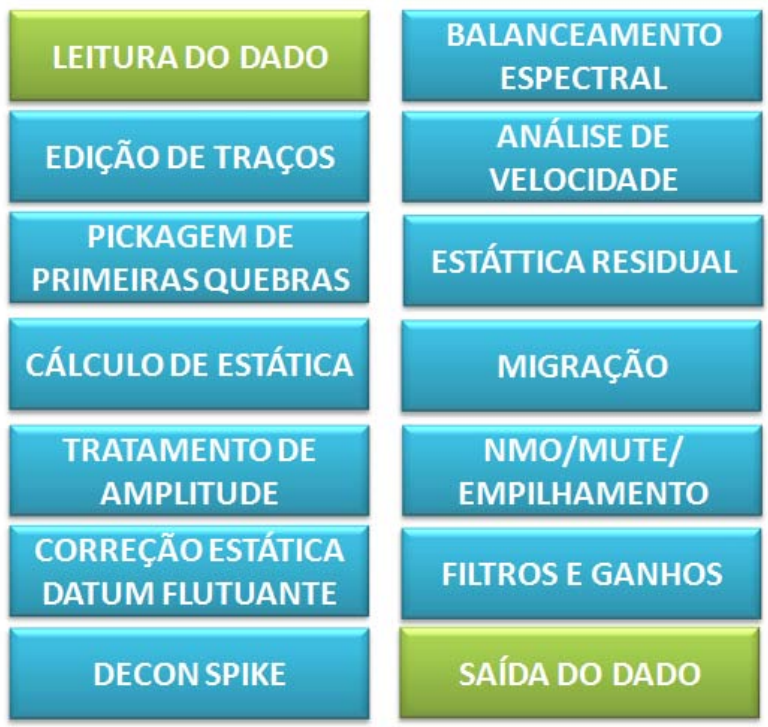

Figura 11 - Fluxo básico do processamento. 
O fluxo do processamento pode ser bem mais completo e complexo do que o apresentado, porém para a finalidade deste trabalho o mesmo poderá se mostrar adequado.

Os dados inicialmente foram submetidos às etapas preliminares de pré-processamento, tais como geometria, edição de traços ruídos, silenciamento (mute) e picagem de primeiras quebras. Após essa etapa iniciamos o processamento propriamente dito, com o cálculo de valores para correção estática, seguido dos processos de tratamento de amplitude, balanceamento espectral, análise de velocidade, cálculo e aplicação da estática residual, empilhamento, filtros e ganhos. Os resultados do processamento da linha testes 2 é mostrado na figura 15.

A picagem das primeiras quebras levou em consideração o offset máximo de 1000 metros. A figura 12 exemplifica um registro picado.

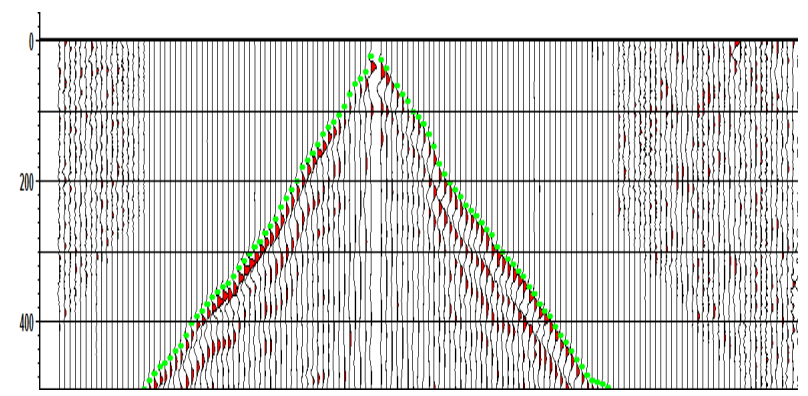

Figura 12 - Picagem de primeiras quebras até offset de $1000 m$.

A figura 13 mostra os pontos de controle para o cálculo da correção estática.

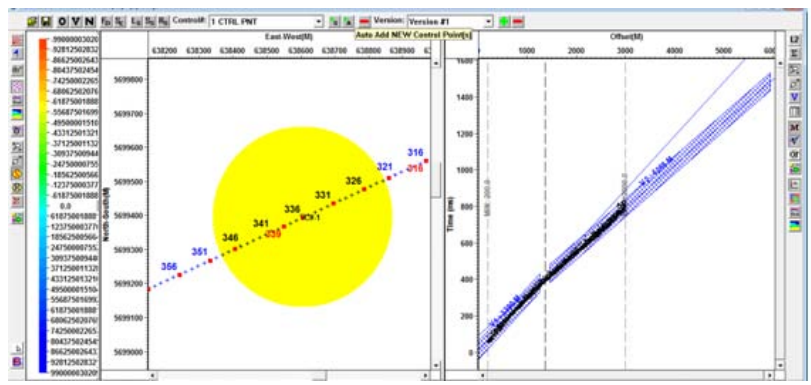

Figura 13 - Cálculo da correção estática.

Após estas etapas os dados foram submetidos à etapa de tratamento de amplitude, onde durante as fases de testes foram possíveis observar resultados bem significativos para a linha teste 2 (arranjo com 2 cargas).

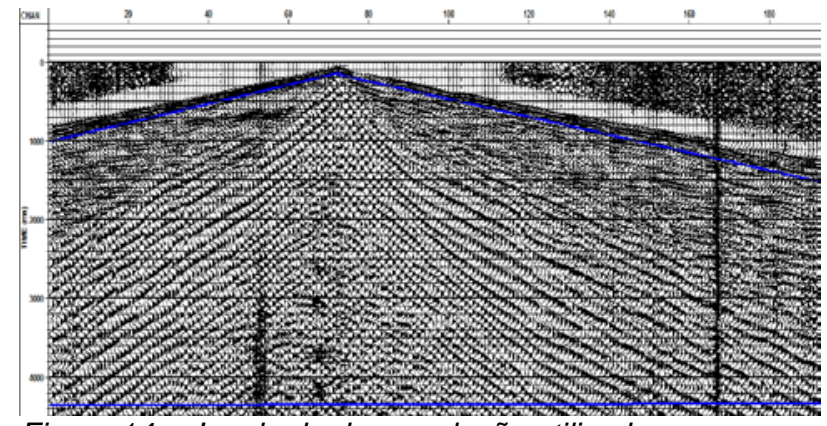

Figura 14 - Janela de deconvolução utilizada.

Então foram submetidos os dados da linha testes 2 às demais etapas do fluxo de processamento como análise de velocidade, estática residual, empilhamento, filtros e ganhos, resultando ao final uma seção sísmica de boa qualidade que embasa a idéia de que o uso do arranjo de cargas 2 apresenta uma resposta satisfatória na relação sinal/ruído.

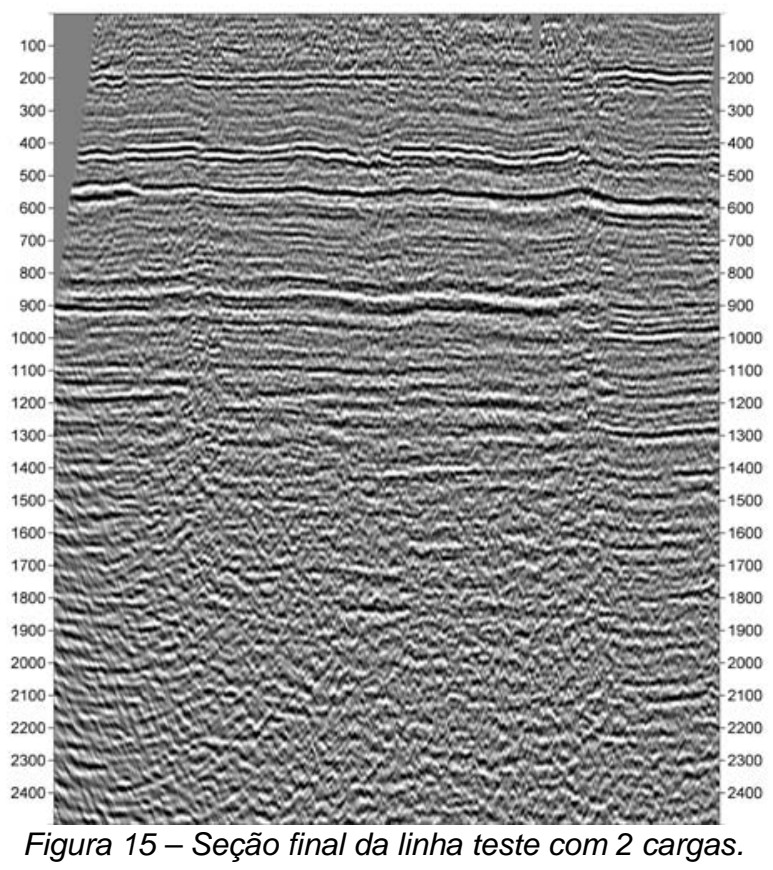

\section{Conclusão}

A análise do sinal sísmico gerado pelos dois arranjos utilizados, nos levou a concluir preliminarmente que os testes realizados indicaram uma melhor relação sinal/ruído para o arranjo de cargas 2, mesmo na faixa de frequência do groundroll, feito que foi comprovado com o resultado obtido após o processamento da linha teste 2 .

\section{Agradecimentos}

Agradecemos a OGX e a Petra Energia por nos fornecer os dados para o presente estudo. Agradecemos a Georadar Levantamentos Geofísicos S/A pelo seu 
suporte técnico. Agradecemos aos profissionais das empresas citadas pelo apoio financeiro e teórico.

\section{Referências}

Pritchett, W. C. 1990, Acquiring better seismic data. Published in the USA by Chapman and Hall. New York NY.

Romanelli, A. L. R. 2010, Análise do sinal sísmico. Sociedade Brasileira de Geofísica (SBGf). Rio de Janeiro.

Vermeer, Gijs J. O. 2011, 3-D Seismic Survey Design. Ed. Society of Exploration Geophysicists, USA, vol. 12.

Yilmaz, O. 2000, Seismic data analysis, in cooper, m. r. and doherty, s. m., ed., seismic data analysis vol. 1, 01 : SEG. 